\title{
Quantitative edge illumination x-ray phase contrast tomography
}

\author{
Charlotte K. Hagen*a, Paul C. Diemoz ${ }^{\mathrm{a}}$, Marco Endrizzi ${ }^{\mathrm{a}}$, Luigi Rigon ${ }^{\mathrm{b}, \mathrm{c}}$, Diego Dreossi ${ }^{\mathrm{d}}$, Fulvia \\ Arfellib,c $^{\mathrm{b}}$, Frances C.M. Lopez ${ }^{\mathrm{b}, \mathrm{c}}$, Renata Longo ${ }^{\mathrm{b}, \mathrm{c}}$ and Alessandro Olivo ${ }^{\mathrm{a}}$ \\ ${ }^{\mathrm{a} D e p a r t m e n t ~ o f ~ M e d i c a l ~ P h y s i c s ~ a n d ~ B i o e n g i n e e r i n g, ~ U n i v e r s i t y ~ C o l l e g e ~ L o n d o n, ~ M a l e t ~ P l a c e, ~}$ \\ Gower Street, London WC1E 6BT, UK, ${ }^{b}$ Department of Physics, University of Trieste, Via Valerio \\ 2, 34127 Trieste, Italy, ${ }^{c}$ Istituto Nazionale di Fisica Nucleare - Sezione de Trieste, Via Valerio 2, \\ 34127 Trieste, Italy, ${ }^{\mathrm{d}}$ Sincrotrone Trieste SCpA, S.S. 14 km 163.5, 34012 Basovizza (TS), Italy
}

\begin{abstract}
This article discusses two experimental setups of edge illumination (EI) x-ray phase contrast imaging (XPCi) as well as the theory that is required to reconstruct quantitative tomographic maps using established methods, e.g. filtered back projection (FBP). Tomographic EI XPCi provides the option to reconstruct volumetric maps of different physical quantities, amongst which are the refractive index decrement from unity and the absorption coefficient, which can be used for dual-mode imaging. EI XPCi scans of a custom-built wire phantom using synchrotron and x-ray tube generated radiation were carried out, and tomographic maps of both parameters were reconstructed. This article further discusses the theoretical basis for the tomographic reconstruction of images showing combined phase and attenuation contrast. Corresponding experimental results are presented.
\end{abstract}

Keywords: X-ray Phase Contrast Imaging, Tomography, Edge Illumination Phase Contrast, Quantitative Imaging, Laboratory-based Phase Imaging

\section{INTRODUCTION}

There has been a steep increase in scientific interest in x-ray phase contrast imaging (XPCi), especially from researchers within biomedical disciplines. The reason is that XPCi can provide images with improved quality compared to standard radiographic methods [1]. In XPCi, contrast arises from phase (refraction) effects, instead of or in addition to attenuation effects, which are exploited in conventional radiography. Both effects can be described by the complex refractive index of the imaged material:

$$
n(E)=1-\delta(E)+i \beta(E)
$$

The parameters $\delta$ and $\beta$ describe the phase shifting and attenuating properties of the material, and $E$ is the photon energy. For weakly attenuating samples and within the energy range used for biomedical imaging, the refractive index decrement $\delta$ can be up to three orders of magnitude larger than the absorption coefficient $\beta$, which explains why XPCi has achieved superior contrast for a range of soft tissue specimens [1].

While most XPCi methods were originally developed at synchrotrons [2-6], a few methods now exist that are compatible also with commercial $\mathrm{x}$-ray equipment which can be found in standard research laboratories [7-11]. This compatibility is crucial to ensure a widespread exploitation of XPCi, especially to make its benefits accessible to a larger group of researchers. One of the methods working with standard equipment, edge illumination (EI) XPCi, has undergone rapid development over the past years $[8,12-18]$ and can now be used not only as a planar imaging modality but also as a computed tomography (CT) one, enabling full 3D imaging [19, 20]. This article summarizes the technical and theoretical requirements to perform tomographic EI XPCi scans. Two different experimental setups ("single slit" and "multi slit" implementations) are briefly discussed; one of them is typically used at synchrotrons and the other one in standard laboratories. The theory for dual-mode quantitative imaging through the reconstruction of tomographic maps of $\delta$ and $\beta$ is provided. Moreover, a formula is presented which allows the reconstruction of tomographic maps showing a combination of phase and attenuation contrast. Experiments were carried out with both synchrotron and laboratory-based EI XPCi setups, and results from both are presented. 


\section{MATERIALS AND METHODS}

\section{1 "Single slit" and "multi slit" implementations}

EI XPCi was initially developed in "single slit" mode [12], the working principle of which is schematically shown in Fig. 1. A laminar beam, shaped by a pre-sample mask, passes through the sample, and, after a distance $z_{\text {od }}$ (object-todetector distance), impinges on a detector. A second mask (detector mask), positioned in front of the detector, covers part of the pixels' surface, hence creating an insensitive area. When the detector mask is positioned in such a way that part of the laminar beam hits the insensitive area and the other part hits the pixels, the edge illumination condition is achieved. In this way, sensitivity towards phase (refraction) effects is achieved in addition to attenuation effects: refraction of the beam towards positive/negative angles results in a higher/lower measured intensity. Changing the relative position of the beam and the detector/mask assembly such that part of the beam falls onto the complementary insensitive area [Fig. 1(b)] has the effect of inverting the refraction contrast. In the "single sit" implementation, the sample has to be scanned along the y-axis to build up a two-dimensional image. For tomographic imaging, the rotation of the sample is also required [19]. Fast scans with the "single slit" implementation are feasible when the x-ray flux is sufficiently high. For this reason, this implementation is typically used for synchrotron-based imaging studies.

a)

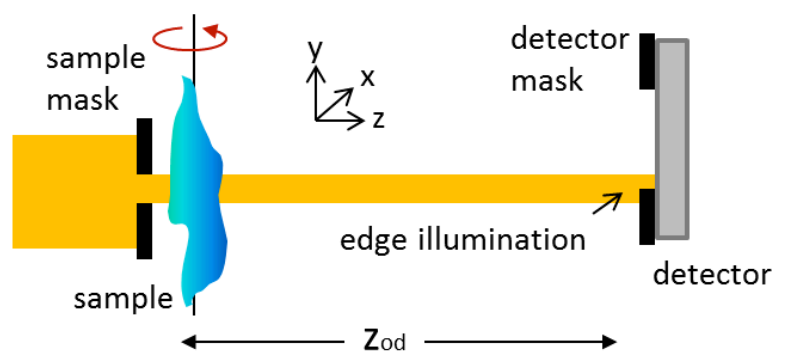

b)

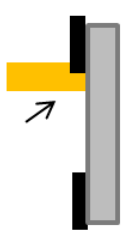

Figure 1. "Single slit" implementation of edge illumination x-ray phase contrast imaging, schematically showing opposing edge illumination conditions [(a) and (b)]. Rotation of the sample enables tomographic imaging. The schematic extends into the plane of the drawing and is not to scale.

In order to enable the single-shot acquisition of two-dimensional images, a second, "multi slit" EI XPCi implementation was developed, the working principle of which is shown in Fig. 2. By means of extended masks, the edge illumination principle is extended to large fields of view and the need to scan the sample is eliminated by replicating the single slit configuration for multiple rows or columns of pixels [8]. The two masks are again placed one upstream of the sample (sample mask) and one before the detector (detector mask). The detector mask, whose projected period matches the pixel size, creates insensitive areas between the pixels. The sample mask, whose projected period also matches the pixel size (in case of a divergent beam, demagnification has to be taken into account), splits the incoming beam into an array of separate beamlets. The gaps between the beamlets are sufficiently large as to prevent interference between them from occurring. When the relative position between the sample mask and the detector/mask assembly is chosen such that each of the beamlets hits the edges of the detector mask (i.e. falls between insensitive and sensitive areas), the edge illumination condition is achieved for each row or column of the area detector. By shifting the beamlets such that they partially fall onto the opposing insensitive areas [Fig. 2(b)], the edge illumination condition, and hence the refraction contrast, is reversed. In the "multi slit" implementation no object scanning is needed to build up a two-dimensional image, hence this is typically used for laboratory-based imaging applications, where x-ray flux is limited due to the use of standard x-ray tubes. Please note that the "multi slit" implementation, like the "single slit" one, fully tolerates divergent cone beams, provided the system magnification is taken into account in the mask design. Tomographic acquisitions require a sample rotation by at least 180 degrees [20]. 
a)

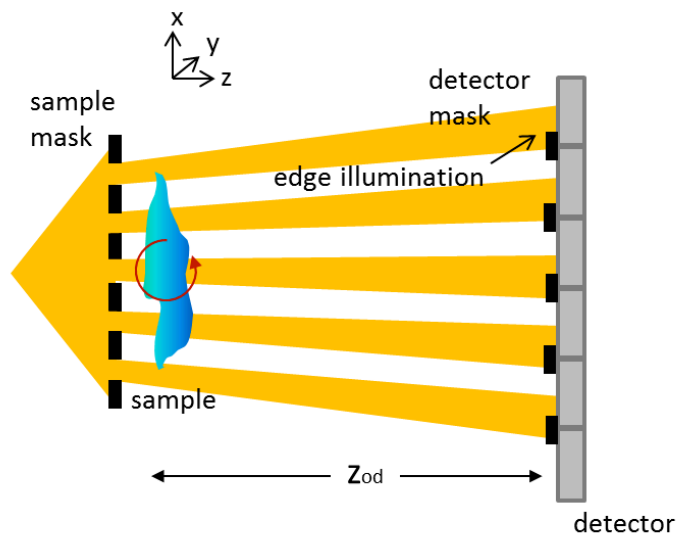

b)

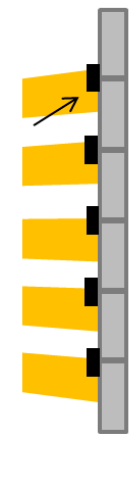

Figure 2. "Multi slit" implementation of edge illumination $x$-ray phase contrast imaging, schematically showing opposite edge illumination conditions [(a) and (b)]. The schematic extends into the plane of the drawing and is not to scale.

The "single slit" and "multi slit" EI XPCi implementations are sensitive to phase (refraction) effects in the plane orthogonal to the masks' slits only. The orientation of the masks (slits in either horizontal or vertical direction) is usually determined on the basis of whether the source is smaller in the horizontal or vertical direction: the smaller the source, the higher the potential angular sensitivity [15]. Moreover, as will be explained in section 2.2, the direction of phase sensitivity determines which physical quantity of the sample can be reconstructed in a CT image. Figures 1 and 2 show different directions of phase sensitivity, as indicated by the different frames of reference: the system in Fig. 1 is sensitive in the vertical (y-) direction, whereas the system in Fig. 2 is sensitive in the horizontal (x-) direction. Systems sensitive to phase effects in two directions simultaneously have been previously investigated [21], and are currently under further development.

The sampling rate of an image acquired with a "single slit" system is determined by the scanning step (along the direction of phase sensitivity) and by the pixel size (in the direction orthogonal to phase sensitivity). In a "multi slit" system, the sampling rate in both directions is determined by the (demagnified) pixel size. However, in the direction of phase sensitivity, it can be artificially increased by dithering, i.e. by shifting the sample by sub-pixel amounts and taking an image at each position, all of which are then combined into a single, highly sampled image [20]. The sampling rate in a dithered image is given by the number of sample displacements (dithering steps) divided by the demagnified pixel size.

\subsection{Tomographic reconstruction}

In both the "single slit" and "multi slit" configuration, the measured intensity on the detector is a function of the relative position of the beam or beamlets and the mask/detector assembly. The corresponding so-called illumination curve, in the following denoted as $C(r)$, takes on its maximum (100\% illumination) when the beam or beamlets fall at the centre of the pixels and its minimum ( $0 \%$ illumination) when the beam or beamlets fall entirely on the solid septa of the mask. The variable $r$ indicates the relative position of the beam or beamlets and the mask/detector assembly. Depending on the direction of phase sensitivity, $r$ refers to either the $\mathrm{x}$ - or the $\mathrm{y}$-axis of the system. The intensity measured on the detector for a fixed relative position $r_{0}$ is hence given by $I(x, y)=I_{0} C\left(r_{0}\right)$, where $I_{0}$ is the intensity measured at $100 \%$ illumination. When a sample is placed in the beam, the intensity becomes $[14,15]$ :

$$
I(x, y)=I_{0} T(x, y) C\left(r_{0}+D(x, y)\right)
$$

where $T$ and $D$ are the transmission of the sample and the refraction-induced displacement of the beam, given by:

$$
T(x, y)=e^{-\mu(x, y)},
$$




$$
D(x, y)=\frac{z_{o d}}{k} \frac{\partial \Phi(x, y)}{\partial r} .
$$

The sample is described in terms of the phase shift $(\Phi)$ and attenuation $(\mu)$ it imposes on the beam:

$$
\begin{aligned}
& \Phi(x, y)=k \int_{l(x, y ; s)} \delta(x, y, z) d s, \\
& \mu(x, y)=2 k \int_{l(x, y ; s)} \beta(x, y, z) d s .
\end{aligned}
$$

The line $l(x, y ; s)$, parameterized by $s$, describes the path of an $\mathrm{x}$-ray hitting the detector plane at $(x, y)$, and $k$ is the wave number. When two images are acquired under opposite edge illumination configurations and processed together according to a dedicated algorithm [16], the attenuation $(\mu)$ and the refraction angle (differential phase), given by:

$$
\alpha(x, y)=\frac{1}{k} \frac{\partial \Phi(x, y)}{\partial r}=\frac{\partial}{\partial r} \int_{l(x, y ; s)} \delta(x, y, z) d s
$$

can be extracted.

When acquired in tomography mode, i.e. when the sample is rotated over a range of at least 180 degrees, separate differential phase and attenuation sinograms can be obtained [19, 20]:

$$
\begin{gathered}
S_{\text {diff.phase }}(x, y ; \theta)=\alpha(x, y ; \theta)=\frac{\partial}{\partial r} \int_{l(x, y ; ; ; \theta)} \delta(x, y, z) d s, \\
S_{a t t}(x, y ; \theta)=2 \underset{l(x, y ; s ; \theta)}{\int_{k} k \beta(x, y, z) d s,}
\end{gathered}
$$

where $\theta$ is the CT rotation angle. From these sinograms, tomographic maps of $\delta$ and $k \beta$ can be reconstructed using standard methods, e.g. filtered back projection (FPB) [22]. The reconstruction of $\delta$ requires an additional integration step along the direction of phase sensitivity prior to or after FBP [19]. The constant of integration can be fixed if an area exists where $\delta$ is constant and known (e.g. air surrounding the sample). When the rotation axis is oriented orthogonal to the direction of phase sensitivity (such as in the setup shown in Fig. 2), $\delta$ can be reconstructed directly from Eq. (8) by employing a specialized filter function in the FBP, which incorporates the integration step [23]. When the $\mathrm{x}$-ray beam is monochromatic, a tomographic map of $\beta$ can be obtained by dividing Eq. (9) by the wave number $k$. When the beam is polychromatic, the reconstructed $\delta$ and $k \beta$ maps refer to effective energies, which are, in general, different for phase and attenuation [24].

When the rotation axis is oriented along the direction of phase sensitivity (such as in the setup shown in Fig. 1), it is not strictly necessary to extract the attenuation and the refraction angle prior to CT reconstruction. From Eq. (2) it is possible to derive a linear relationship between the measured intensity and the sample attenuation and induced refraction angle $[19,25]$. Applying the logarithm to both sides of Eq. (2) yields, in fact:

$$
\ln \left(\frac{I(x, y)}{I_{0} C\left(r_{0}\right)}\right)=-\mu(x, y)+\ln \left(C\left(r_{0}+z_{o d} \alpha(x, y)\right)\right)-\ln \left(C\left(r_{0}\right)\right) .
$$

To be suitable for $\mathrm{CT}$ reconstruction, the terms on the right must be expressible as line integrals. While the attenuation $\mu$ is a line integral by definition and $\ln \left(C\left(r_{0}\right)\right)$ is a constant, the second term requires a linearization around $r_{0}$ [25]:

$$
\ln \left(C\left(r_{0}+z_{o d} \alpha(x, y)\right)\right) \approx \ln \left(C\left(r_{0}\right)\right)+\frac{C^{\prime}\left(r_{0}\right)}{C\left(r_{0}\right)} z_{o d} \alpha(x, y),
$$


which is valid if $r_{0}$ corresponds to the linear part of the illumination curve $C$. Further, the approximation is valid for small refraction angles such as encountered in most biomedical applications and under the assumption of small scattering angles. Inserting Eq. (11) into Eq. (10) yields:

$$
\ln \left(\frac{I(x, y)}{I_{0} C\left(r_{0}\right)}\right)=-\mu(x, y)+A \cdot \alpha(x, y)
$$

where $A=z_{\text {od }} C^{\prime}\left(r_{0}\right) / C\left(r_{0}\right)$ is a factor that depends only on the imaging system. When acquired in CT mode, Eq. (12) becomes a sinogram of the form:

$$
S_{\text {mixed }}(x, y ; \theta)=-\frac{1}{2} \ln \left(\frac{I(x, y ; \theta)}{I_{0} C\left(r_{0}\right)}\right)=\int_{l(x, y ; s ; \theta)}\left(k \beta(x, y, z)-\frac{A}{2} \cdot \frac{\partial}{\partial r} \delta(x, y, z)\right) d s .
$$

From Eq. (13), tomographic "mixed" attenuation and differential phase maps can be reconstructed with FBP [19].

\subsection{Experiments}

Two sets of experiments were carried out to validate the theory. During the first experiment, a custom-built wire phantom was scanned with a "single slit" EI XPCi setup at the SYRMEP (Synchrotron Radiation for Medical Physics) beamline of the Elettra synchrotron (Trieste, Italy) [26]. During the second experiment, the same phantom was scanned with a laboratory-based "multi slit" EI XPCi setup implemented with conventional, off-the-shelf x-ray equipment. The custom-built phantom consisted of five wires (1. Nylon 6, diam. $150 \mu \mathrm{m} \pm 20 \%$, 2. Polyether ether ketone (PEEK), diam. $450 \mu \mathrm{m} \pm 20 \%$, 3. Polybutylene terephthalate (PBT), diam. $180 \mu \mathrm{m} \pm 20 \%$, 4. Sapphire, diam. $250 \mu \mathrm{m} \pm 20 \%, 5$. Titanium, diam. $250 \mu \mathrm{m} \pm 10 \%$ ), which were tilted with respect to the vertical axis. For the sake of generality, the materials were chosen to range from weakly to highly attenuating / refracting. The diameters are the ones specified by the supplier (Goodfellow Inc., UK).

During the synchrotron experiment, a Huber slit (Huber GmbH, Germany) with an opening of $20(\mathrm{H}) \mathrm{x}_{0.02}(\mathrm{~V}) \mathrm{mm}^{2}$ was used as pre-sample mask. The slit was placed at approximately $22 \mathrm{~m}$ from the source, which has full width at half maximum dimensions of $0.28(\mathrm{H}) \times 0.08(\mathrm{~V}) \mathrm{mm}^{2}$. The "PICASSO" single-photon counting Si strip detector, developed by the Istituto Nazionale di Fisica Nucleare (INFN, Italy) and based on the Mythen ASIC [27-29], with a 210 (H) x 0.3 (V) $\mathrm{mm}^{2}$ active surface and pixel size of $50(\mathrm{H}) \times 300(\mathrm{~V}) \mu \mathrm{m}^{2}$ was located at approximately $0.9 \mathrm{~m}$ downstream of the sample slit. A tungsten slit thick enough to absorb all x-rays at the used energy, was used as detector mask. The objectto-detector distance was $0.7 \mathrm{~m}$. The beam energy was set to $20 \mathrm{keV}$ with a fractional bandwidth of $0.2 \%$ by a double crystal Si $(1,1,1)$ monochromator.

The projections were acquired under complimentary edge illumination conditions [Figs 1(a) and 1(b)] with an exposure time of $0.1 \mathrm{~s}$. The sample was rotated over a 180 degree range with an angular step of 0.5 degrees. Moreover, it was scanned vertically with a $5 \mu \mathrm{m}$ scanning step. Sinograms in the form of Eqs. (8) and (9) were generated according to reference [16], from which tomographic maps of $\delta$ and $k \beta$ were reconstructed with FBP. For the reconstruction of the $\delta$ map, an additional integration step was included to convert differential into absolute values [19]. The constant of integration was fixed by assuming that $\delta=0$ for the air surrounding the wires. Although a monochromatic beam was used, the division of the $k \beta$ map was neglected in order to obtain values of the same order of magnitude as from the (polychromatic) laboratory-based experiment described below. In addition to separate differential phase and attenuation sinograms, mixed differential phase and attenuation sinograms in form of Eq. (13) were generated directly from the projections acquired under only one of the edge illumination conditions [Fig. 1(a)]. Again, FBP was used for CT reconstruction. In order to validate the relationship predicted by Eq. (13), a linear combination of the separately reconstructed differential phase and attenuation maps available from the previous step was computed using appropriate scaling factors $k$ and $A / 2$ for comparison.

During the laboratory-based experiment, the Rigaku MicroMax $007 \mathrm{HF}$ rotating anode (molybdenum) x-ray tube (Rigaku Corporation, Japan) was employed. The source was operated at $35 \mathrm{kVp}$ and $25 \mathrm{~mA}$, which corresponds to an energy spectrum with a mean energy of $18 \mathrm{keV}$. The size of the source's focal spot was measured to be approximately 70 
$\mu \mathrm{m}$ horizontally. The detector was the Hamamatsu C9732DK flat panel, a passive-pixel CMOS sensor with a pixel size of $50 \times 50 \mu \mathrm{m}^{2}$. The detector was located at $2 \mathrm{~m}$ from the source. The two x-ray masks, fabricated by electroplating gold strips onto graphite substrates, were located at $1.6 \mathrm{~m}$ (sample mask) and $1.96 \mathrm{~m}$ (detector mask) from the source. The masks' periods were $79 \mu \mathrm{m}$ (sample mask) and $98 \mu \mathrm{m}$ (detector mask), and their slits were $23 \mu \mathrm{m}$ and $29 \mu \mathrm{m}$ wide respectively. With these dimensions, every second pixel column was covered ("line-skipping" configuration [30]), which reduces the effect of cross-talk between the pixels. The object was positioned $5 \mathrm{~cm}$ downstream of the sample mask.

Tomographic acquisitions were carried out over an angular range of 360 degrees with an angular step of 0.5 degrees. At each rotation angle, two projections were acquired under opposing edge illumination conditions, each with six dithering steps (displacement of the object in x-direction by a sixth of the sample mask period). From the projections, sinograms in form of Eq. (8) and (9) were created according to reference [16]. Tomographic maps of $\delta$ and $k \beta$ were reconstructed with FBP. For the $\delta$ maps, the Hilbert filter was used instead of the standard ramp filter in order to account for the derivative operator in Eq. (8) [23]. It should be noted that, because the full energy spectrum of the X-ray tube was used for imaging, the reconstructed maps refer to effective energies.

In addition to the wire phantom, a biological object (a dung beetle) was imaged with the laboratory-based setup. The scanning parameters and acquisition procedure were the same as described above.

\section{RESULTS}

The image in Fig. 3(a) shows the results of the mixed reconstruction obtained from the synchrotron experiment, the possibility of which was suggested by Eq. (13). The five wires in the custom-built phantom, numbered from 1 to 5, are visible due a low frequency area contrast caused by attenuation and high frequency fringes on the contours caused by refraction. It should be noted that the area contrast for the weakly attenuating wires (no. 1-3) is almost not present and that these wires are only visible due to phase contrast. The streak artefacts around the titanium wire (no. 5) are due to its comparably very strong attenuation. The contrast was stretched beyond saturation to visualize all wires at the same time. Figure 3(b) shows the linear combination of separately reconstructed attenuation and differential phase slices scaled by the wave number $k$ and a factor of $A / 2=-2.7 \cdot 10^{4}$ respectively, the latter being calculated from the values of the measured illumination curve and its differential at the relative position $r_{0}$ of the beam and mask/detector assembly and from the object-to-detector distance. Visually, Figs. 3(a) and 3(b) are indistinguishable, which validates Eq. (13). This is locally confirmed by Figs. 3(c) and 3(d), which show profiles across the nylon 6 wire (no. 1) and the titanium wire (no. 5) respectively. It should be noted that these are the wires with the weakest and highest attenuation and refraction, and that a good agreement of the profiles can be observed in both cases.

(a) Mixed reconstruction

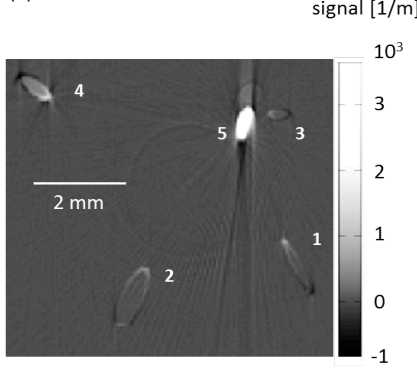

(b) Linear combination

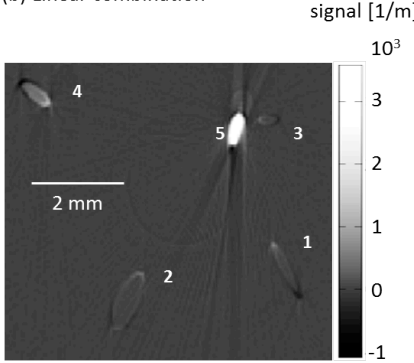

(c)

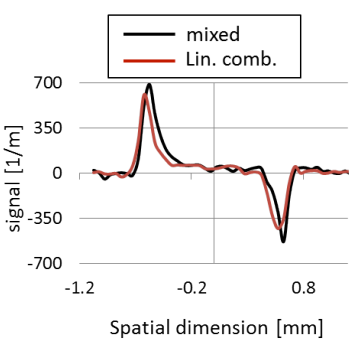

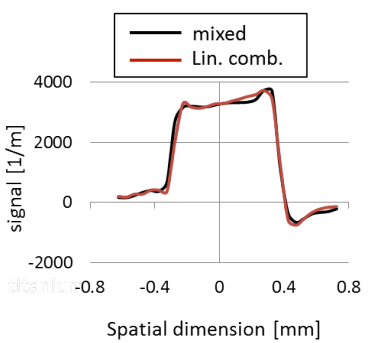

Figure 3. A transverse slice through the reconstructed volume of a custom-built wire phantom (see text for a list of the wire's materials and diameters) showing a mixed attenuation and differential phase contrast (a), the same slice showing the linear combination of separately reconstructed attenuation and differential phase contrast (b) and profiles across the nylon 6 (c) and titanium (d) wires extracted from both maps.

Figures 4(a) and 4(b) show the results of the quantitative analysis of the reconstructed $\delta$ and $k \beta$ maps, obtained from the synchrotron (green triangles) and the laboratory-based experiments (orange circles). The retrieved values of $\delta$ and $k \beta$ within the wires were extracted and plotted against the corresponding wire. In order to extract the values, the 
reconstructed slices were averaged within regions of interest fully contained in the wires. In the synchrotron case, 100 transverse slices (corresponding to a vertical portion of $0.5 \mathrm{~mm}$ of the phantom) were averaged prior to extraction. All error bars in the plots correspond to one standard deviation of all pixels that were averaged to obtain the results.

The plots also contain the nominal $\delta$ and $k \beta$ values of the wires' materials at $20 \mathrm{keV}$ (grey bars), calculated according to reference [31]. In order to account for potential material impurity and for the uncertainty on density, an error of $\pm 5 \%$ has been assigned to the calculated values. As the synchrotron experiment was performed at $20 \mathrm{keV}$, the retrieved values can be directly compared to the nominal ones. It is apparent that the general trend of the retrieved values is correct, however, it can also be seen that a slight under estimation exists. We attribute this to our non-optimal data acquisition scheme: the CT rotation was performed in a continuous mode, i.e. the rotator kept spinning at all times during the acquisition, also during the vertical displacement that was needed due to the "single slit" implementation. This caused an angular offset between adjacent transverse slices, which could only partially be corrected for due to the fact that the angular range covered during the vertical displacement was not exactly a multiple of the angular step of the tomographic acquisition. The slight offset manifested as stripe artefacts during the integration step necessary for the reconstruction of the $\delta$ map. These artefacts are the cause for the slightly underestimated retrieved $\delta$ values [19]. In addition, these artefacts explain the large error bars on the retrieved $\delta$ values. It is noteworthy that the range of retrievable $\delta$ values is large, in particular compared to other XPCi techniques [32]. The reason is that the "single slit" implementation does not impose any restriction on the width of the detector mask slit, i.e. on the largest resolvable refraction angle. The retrieved $k \beta$ values shown in Fig. 4(b) match the nominal ones with a reasonable accuracy.

(a)

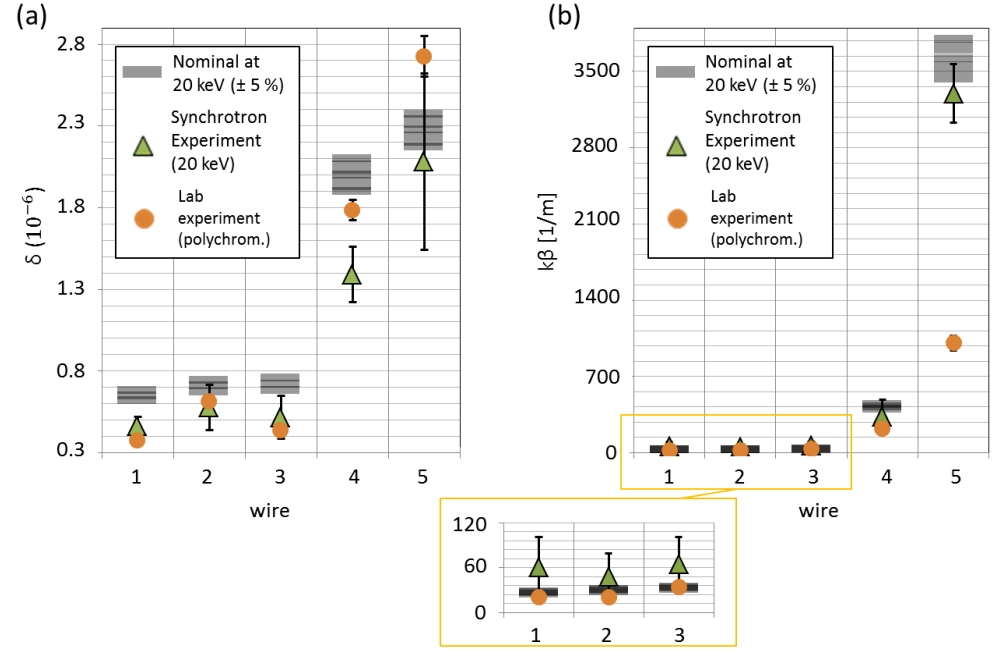

(c)

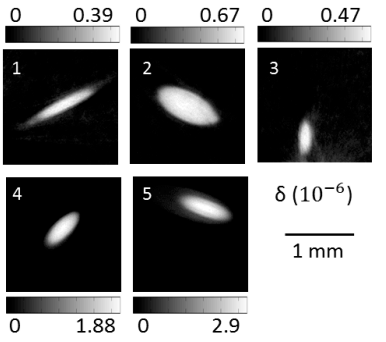

(d) 0

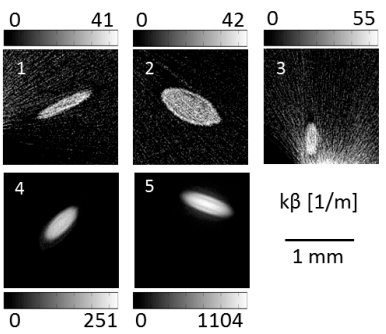

Figure 4. Retrieved $\delta$ (a) and $k \beta$ (b) values for the wires in the phantom (see text for a list of materials and diameters), extracted from data acquired during the synchrotron (green triangles) and lab-based (orange circles) experiments. The plots also contain the nominal $\delta$ and $k \beta$ values of the wires' materials at $20 \mathrm{keV}$, calculated according to reference [31]. A $\pm 5 \%$ uncertainty on the nominal values was assumed. Panels (c) and (d) show the $\delta$ and $k \beta$ maps respectively of the wires reconstructed from the data acquired during the laboratory experiment.

The $\delta$ and $k \beta$ values extracted from the experimental data acquired with the laboratory-based setup (orange circles in Figs. 4(a) and 4(b)) are less straightforward to evaluate since they have been acquired with polychromatic radiation. The mean energy of the spectrum is $18 \mathrm{keV}$, which could lead one to expect retrieved values slightly larger than those retrieved at $20 \mathrm{keV}$. However, instead of the spectrum's mean energy, effective energies have to be considered. These are determined by the imaging system (energy spectrum, detector characteristics and spectral properties of the sample and detector mask), as well as by the imaged object itself (spectral properties and thickness). In references [20] and [24], it has been demonstrated that the effective energies, and, hence, the expected $\delta$ and $k \beta$ values, can be calculated accurately if all these parameters are known. In order to compare the $\delta$ and $k \beta$ values retrieved from the lab-based 
experiment to theoretical values, one would need to have an accurate knowledge of, among other things, the spectral detector response, which requires a thorough detector characterization. Since the detector that was used for this experiment had not yet been adequately characterized, a comparison of the retrieved with theoretical $\delta$ and $k \beta$ values was not possible. For a comparison between retrieved and theoretical $\delta$ and $k \beta$ values which were acquired with a similar labbased "multi silt" EI XPCi setup but with a detector that was fully characterized, the reader is referred to a previous publication [20]. However despite not being able to perform a theoretical analysis of the retrieved $\delta$ and $k \beta$ values, it should be noted that they follow a sensible trend for all materials, with the $k \beta$ value retrieved for the titanium wire (no. 5) being the only one that seems underestimated. However, titanium lies outside the range of materials generally encountered in biomedical imaging applications due to its very strong attenuation. It should again be noted that the range of retrievable $\delta$ values is large. Although in the "multi slit" implementation an upper limit to the largest resolvable refraction angle exists, and is effectively given by the width of the detector mask slits [17], this is much larger than for other XPCi methods [32].

As an example of tomographic maps of the wire phantom, Figures 4(c) and 4(d) show the $\delta$ and $k \beta$ maps reconstructed from the data acquired during the laboratory experiment. Although all wires were scanned simultaneously, they are shown in different windows in order to allow for an appropriate grey value windowing. The strong artefact at the bottom of the $k \beta$ map of the PBT wire (no. 3) originates from the fact that this wire was located close to the titanium wire during the scan (see also Fig. 3). The strong attenuation of titanium causes the observed streak artefact. This artefact is not present in the corresponding $\delta$ map, as the relative difference in $\delta$ between PBT and titanium is smaller.

Figure 5 shows the results of the laboratory-based scan of a biological object (a dung beetle): in particular a tomographic $\delta$ map across its abdomen, the corresponding $k \beta$ map and a volume rendering of the full $\delta$ map are shown. The volume rendering was created with the open-source software 3DSlicer (www.slicer.org). Fine features like the hairs on the beetle's leg can be appreciated when zooming closer into the volume [Fig. 5(d)]. Moreover, the zoom of a feature in Figs. 5(a) and 5(b) shows a higher contrast on a lower noise background (superior contrast-to-noise ratio) in the $\delta$ map compared to the $k \beta$ map. In a previous publication it was shown that 3D EI XPCi images of similar quality as those shown in Fig. 5 can be acquired with a radiation dose of a few tens of mGy [20].
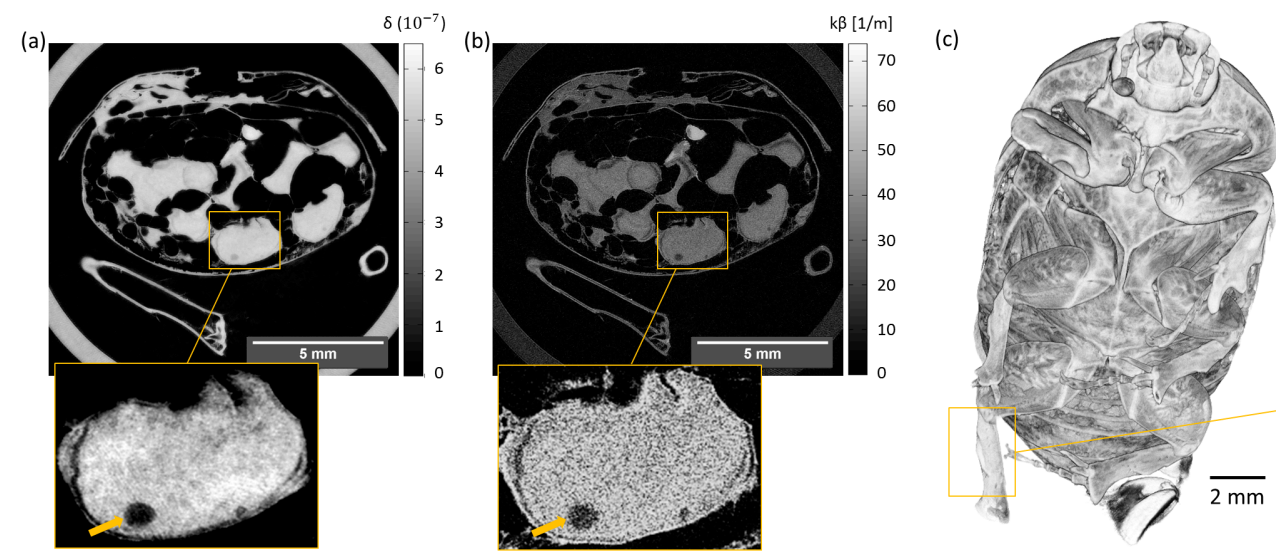

(d)

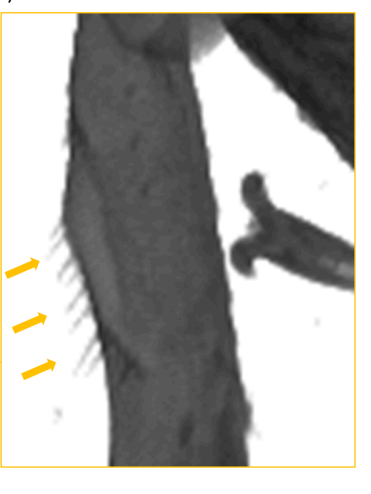

Figure 5. Tomographic $\delta$ (a) and $k \beta$ (b) maps showing a transverse cross-section through the abdomen of a dung beetle, and a volume rendering of the full $\delta$ map (c) as well as a zoom around the beetle's leg (d).

\section{CONCLUSION}

A description of two experimental setups ("single slit" and "multi slit" implementations) and the theory to perform quantitative tomographic EI XPCi scans were provided. Three different types of tomographic maps can be reconstructed, showing the refractive index decrement $\delta$, the absorption coefficient (times the wave number) $k \beta$, and a linear combination of the attenuation and differential of $\delta$. While the reconstruction of the former two maps requires the separation of phase and attenuation contrast, the latter can be reconstructed directly from the projections, even when only 
one projection is acquired at each rotation angle. The mixed reconstruction requires the data to be taken with an EI XPCi setup in which the direction of phase sensitivity coincides with the orientation of the rotation axis. The separate $\delta$ and $k \beta$ maps can be obtained also with a setup in which the direction of phase sensitivity is orthogonal to the orientation of the rotation axis.

Two sets of experiments have been performed to validate the theory: one with a "single slit" EI XPCi setup at a synchrotron, and one with a "multi slit" setup implemented entirely with commercial x-ray equipment. The phantom was custom-built and contained a set of wires of known materials. The $\delta$ and $k \beta$ maps reconstructed from the synchrotron data could be compared to nominal values, and the expected trend could be observed in the retrieved values. Small deviations of the retrieved $\delta$ values from the nominal ones could be attributed to problems during the experimental acquisition. For the maps originating from the laboratory-based experiment, a direct comparison was not possible due to the polychromatic spectrum of the x-ray tube and unknown spectral detector characteristics. However, the reconstructed values again followed the expected trend. The possibility to obtain a precise match with the predictions of an "effective energy" model [24] was demonstrated previously [20].

The reconstruction of separate $\delta$ and $k \beta$ maps is the basis for truly quantitative imaging, i.e. material identification on the basis of the grey values in the images. In this sense, EI XPCi provides the option for dual-mode imaging: an unknown material can be characterized via its attenuating and phase shifting properties. On the other hand, the mixed reconstruction approach can often be appropriate for samples where it is sufficient to see the contours of features within the object. This approach has the advantage that no separation of phase and attenuation contrast is required, which means that the acquisition of a single projection per rotation angle is sufficient. Consequently, this reconstruction approach is well suited to applications in which the minimization of radiation dose and scan time is crucial.

\section{ACKNOWLEDGMENTS}

This work was supported by the UK Engineering and Physical Sciences Research Council (Grant Nos. EP/G004250/1, EP/I021884/1 and EP/L001381/1). P.C.D. and M.E. are supported by Marie Curie Career Integration Grant Nos. PCIG12-GA-2012-333990 and PCIG12-GA-2012-334056 within the Seventh Framework Programme of the European Union.

*charlotte.hagen.10@ucl.ac.uk

\section{REFERENCES}

[1] Lewis, R., Hall, C., Hufton, A., Evans, S., Menk, R., Arfelli, F., Rigon, L., Tromba, G., Dance, D., Ellis, I., Evans, A., Jacobs, E., Pinder, S. and Rogers, K., "X-ray refraction effects: Application to the imaging of biological tissues," Br. J. Radiol. 76, 301-308 (2003).

[2] Momose, A. and Fukuda, J, "Phase contrast radiographs of nonstained rat cerebellar specimen," Med. Phys. 22(4), 375-379 (1995).

[3] Snigirev, A., Snigireva, I., Krohn, V., Kuznetsov, S. and Schelokov, I., "On the possibilities of x-ray phase contrast microimaging by coherent high energy synchrotron radiation," Rev. Sci. Instrum. 66, 5486-5492 (1995).

[4] Ingal, V.N. and Beliaevskaya, E.A., "X-ray plane-wave topography observation of the phase contrast from a non-crystalline object," Journal of Physics D: Applied Physics 28, 2314-2317 (1995).

[5] Momose, A., Takeda, T., Itai, Y. and Hirano, K., "Phase-contrast X-ray computed tomography for observing biological soft tissues," Nature Medicine 2(4), 473-475 (1996).

[6] Chapman, D., Thomlinson, W., Johnston, R.E., Washburn, D., Pisano, E., Gmur, N., Zhong, Z., Menk, R., Arfelli, F. and Sayers, D., "Diffraction enhanced x-ray imaging," Phys. Med. Biol. 42, 2015-2025 (1997).

[7] Pfeiffer, F., Weitkamp, T., Bunk, O. and David, C., "Phase retrieval and differential phase contrast imaging with low-brilliance x-ray sources," Nature Physics 2, 285-261 (2006). 
[8] Olivo, A. and Speller, R.D., "A coded-aperture technique allowing x-ray phase contrast imaging with conventional sources," Applied Physics Letters 91, 074106 (2007).

[9] Bevins, N., Zambelli, J., Li, K., Qi, Z. and Chen, G., "Multicontrast X-ray computed tomography imaging using Talbot-Lau interferometry without phase stepping," Med. Phys. 39(1), 424-428 (2012).

[10] Wen, H., Bennett, E.E., Hegedus, M.M. and Rapacchi, S., "Fourier x-ray scattering radiography yields bone structural information," Radiology 251(3), 910-918 (2010).

[11] Parham, C., Zhong, Z., Connor, M., Chapman, D. and Pisano, E., "Design and implementation of a compact low-dose diffraction enhanced medical imaging system," Acad. Radiol. 16(8), 911-917 (2009).

[12] Olivo, A., Arfelli, F. Cantatore, G., Longo, R., Menk, R., Pani, S., Prest, M., Poporat, P., Rigon, L., Tromba, G., Vallazza, E. and Castelli, E., "An innovative digital imaging set-up allowing a low-dose approach to phase contrast applications in the medical field," Med. Phys. 28(8), 1610-1619 (2001).

[13] Millard, T.P., Endrizzi, M., Ignatyev, K., Hagen, C.K., Munro, P.R.T., Speller, R. and Olivo, A., "Method for the automatization of the alignment of a laboratory based x-ray phase contrast edge illumination system", Rev. Sci. Instrum. 84, 083702 (2013).

[14]Diemoz, P.C., Endrizzi, M., Zapata, C.E., Pesic, Z., Rau, C., Bravin, A., Robinson, I. and Olivo, A., "X-ray phase contrast imaging with nanoradian angular resolution," Physical Review Letters 110(13), 138105 (2013).

[15] Diemoz, P.C., Hagen, C.K., Endrizzi, M. and Olivo, A., "Sensitivity of laboratory based implementations of edge illumination x-ray phase contrast imaging," Applied Physics Letters 103, 244104 (2013)

[16] Munro, P.R.T., Hagen, C.K., Szafraniec, M.B. and Olivo, A., "A simplified approach to quantitative coded aperture x-ray phase imaging," Optics Express 21(9), 11187-11201 (2013).

[17] Munro, P.R.T., Rigon, L., Ignatyev, K., Lopez, F.C.M., Dreossi, D., Speller, R.D. and Olivo, A., "A quantitative, non-interferometric x-ray phase contrast imaging technique," Optics Express 21(1), 647-661 (2013).

[18] Diemoz, P.C., Vittoria, F.A. and Olivo, A., "Spatial resolution of edge illumination x-ray phase contrast imaging," Optics Express 22(13), 15514-15529 (2014).

[19] Hagen, C.K., Diemoz, P.C., Endrizzi, M., Rigon, L., Dreossi, D., Arfelli, F., Lopez, F.C.M., Longo, R. and Olivo, A., "Theory and preliminary experimental verification of quantitative edge illumination x-ray phase contrast tomography," Optics Express 22(7), 7989-8000 (2014).

[20] Hagen, C.K., Munro, P.R.T., Endrizzi, M., Diemoz, P.C. and Olivo, A., "Low-dose phase contrast tomography with conventional x-ray sources," Med. Phys. 41(7), 070701 (2014).

[21] Olivo, A., Bohndiek, S.E., Griffiths, J.A., Konstantinidis, T. and Speller, R.D., "A non-free space propagation $\mathrm{x}$-ray phase contrast imaging method sensitive to phase effects in two directions simultaneously," Applied Physics Letters 94, 044108 (2009).

[22] Kak, A.C. and Slaney, M., [Principles of Computerized Tomographic Imaging], IEEE Press, New York (1988).

[23]Huang, Z., Kang, K., Li, Z., Zhu, P., Yuan, Q., Huang, W., Huang, J., Zhang, D. and Yu, A., "Direct computed tomographic reconstruction for directional derivative projections of computed tomography of diffraction enhanced imaging," Applied Physics Letters 89, 041124 (2006)

[24] Munro, P.R.T. and Olivo, A., "X-ray phase contrast imaging with polychromatic sources and the concept of effective energy," Phys. Rev. A. 87, 053838 (2013).

[25] Diemoz, P.C., Coan, P., Zanette, I., Bravin, A., Lang, S., Glaser, C. and Weitkamp, T., "A simplified approach for computed tomography with an x-ray grating interferometer," Optics Express 19(3), 1691-1698 (2011).

[26] Tromba, G., Longo, R., Abrami, A., Arfelli, F., Astolfo, A., Bregant, P., Brun, F., Casarin, K., Chenda, V., Dreossi, D., Hola, M., Kaiser, J., Manchini, L., Menk, R., Quai, E., Quaia, E., Rigon, L., Rokvic, T., Sodini, N., Sanabor, N., Schultke, E., Tonutti, M., Vascotto, A., Zanconati, F., Cova, M. and Castelli, E., "The SYRMEP beamline of Elettra: clinical mammography and biomedical applications," AIP Conference Proceedings 1266, 18-23 (2010).

[27] Rigon, L., Arfelli, F., Astolfo, A., Bergamaschi, A., Dreossi, D., Longo, R., Menk, R.H., Schmitt, B., Vallazza, E. and Castelli, E., "A single-photon counting "edge-on" silicon detector for synchrotron radiation mammography," Nuclear Instruments and Methods in Physics Research Section A 608(1), S62-S65 (2009).

[28] Lopez, F.C., Rigon, L., Longo, R., Arfelli, F., Bergamaschi, A., Chen, R.C., Dreossi, D., Schmitt, B., Vallazza, E. and Castelli, E., "Development of a fast read-out system of a single photon counting detector for mammography with synchrotron radiation," JINST 6, C12031 (2011). 
[29] Mozzanica, A., Bergamaschi, A., Dinapoli, R., Gozzo, F., Henrich, B., Kraft, P., Patterson, B. and Schmitt, B., "MythenII: a 128 channel single photon counting readout chip," Nuclear Instruments and Methods in Physics Research Section A 607(1), 250-252 (2009).

[30] Ignatyev, K., Munro, P.R.T., Speller, R.D. and Olivo, A., "Effects of signal diffusion on x-ray phase contrast images," Rev. Sci. Instrum 82, 073702 (2011).

[31] Henke, B.L., Gullikson, E.M. and Davis, J.C., "X-ray interactions: photoabsorption, scattering, transmission and reflection at $\mathrm{E}=50-30000 \mathrm{eV}, \mathrm{Z}=1-92, "$ Atomic Data and Nuclear Data Tables 54, 181-342 (1993).

[32]Zhu, P., Zhang, K., Wang. Z., Liu, Y., Liu, X., Wu, Z., McDonald, S.A., Marone, F. and Stampanoni, M., "Low-dose, simple, and fast grating-based $\mathrm{x}$-ray phase-contrast imaging," Proceedings of the National Academy of Sciences USA 107(31), 13576-13581 (2010). 